\title{
EFEITO DA INTERAÇÃO TEMPERATURA-TENSÃO DE ÁGUA SOBRE A GERMINAÇÃO DE MICROESCLERÓDIOS DE Macrophomina phaseolina*
}

\author{
FRANCISCO M. P. VIANA ${ }^{1} \&$ NILTON L. DE SOUZA ${ }^{2}$
}

\begin{abstract}
${ }^{1}$ Centro Nacional de Pesquisa de Agroindústria Tropical (CNPAT), Empresa Brasileira de Pesquisa Agropecuária (Embrapa), Cx. Postal 3761, 60511-110, Fortaleza-CE, e-mail: fmpviana @ cnpat.embrapa.br; ${ }^{2}$ Departamento de Defesa Fitossanitária, UNESP, Cx. Postal 237, 18603-970, Botucatu-SP, e-mail: imorita @ fmb.unesp.br.
\end{abstract}

(Aceito para publicação em 15/03/2002)

Autor para correspondência: Francisco M. P. Viana

VIANA, F.M.P. \& de SOUZA, N.L. Efeito da interação temperatura-tensão de água sobre germinação de microescleródios de Macrophomina phaseolina. Fitopatologia Brasileira 27:268-272. 2002.

\section{RESUMO}

Em regiões tropicais e de temperaturas elevadas, Macrophomina phaseolina é agente de podridão em raízes, caule, ramos, e frutos em mais de 500 espécies de plantas cultivadas. Entretanto, pouco se sabe acerca da influência da temperatura e da umidade sobre a germinação dos microescleródios desse fungo. O objetivo deste trabalho foi estudar o efeito da interação temperatura-tensão de água na areia sobre a germinação dos microescleródios do fungo. Os trabalhos foram conduzidos em condições de laboratório, tendo sido realizados em duas etapas. Em um primeiro experimento, avaliou-se a interação de cinco temperaturas com duas tensões de água. Os resultados obtidos subsidiaram um segundo experimento no qual avaliou-se a interação de duas temperaturas com seis tensões de água no substrato. O delineamento experimental para ambos experimentos foi o inteiramente casualizado com quatro repetições, em esquema fatorial, com arranjo combinatório $5 \times 2$ e $2 \times 6$, respectivamente, para o primeiro e segundo experimentos. Os resultados mostraram $(\mathrm{P} \leq 0,05)$ que os maiores percentuais de germinação ocorreram às temperaturas de $30{ }^{\circ} \mathrm{C}$ e $33{ }^{\circ} \mathrm{C}$. Na tensão de água de $-1.500 \mathrm{kPa}$ ocorreu germinação em todas as temperaturas testadas. A tensão de água de $-80 \mathrm{kPa}$ tendeu a proporcionar o maior percentual de germinação, embora não tenha diferido das tensões de $-8,-300$ e $-1.500 \mathrm{kPa}$. Os microescleródios não germinaram em areia seca ao ar, porém germinaram em $0 \mathrm{kPa}$, embora em menor percentual que a -8 , $80,-300$ e $-1.500 \mathrm{kPa}$. O efeito isolado da tensão de água sobre a germinação foi importante apenas quando se considerou os níveis extremos desse fator.

Palavras-chave adicionais: fungo do solo, potencial matricial, viabilidade.

\section{ABSTRACT \\ Effect of temperature-water tension interaction on germination of Macrophomina phaseolina microsclerotia}

In tropical regions and warm temperature regions, Macrophomina phaseolina is a causal agent of root, stem, and fruit decay in more than 500 cultivated plants species. However, not much is known about the influence of temperature and soil humidity in the germination of microsclerotia. The objective of this study was to evaluate the effect of temperature-water tension interaction in the sand substract on the germination of microsclerotia of the fungus. This work was carried out under laboratory conditions and involved two phases. In the first phase the effect of the interaction of five temperatures with two water tension levels was evaluated. In the second phase, the effect of the interaction of two temperatures with six water tension levels was evaluated. A completely randomized design with factorial arrangement and four replications was used for both experiments. The results showed $(\mathrm{P}<0.05)$ that the greatest percents of germination occurred at $30{ }^{\circ} \mathrm{C}$ and $33{ }^{\circ} \mathrm{C}$. At water a tension of $-1,500 \mathrm{kPa}$ microesclerotia germination occurred at all temperatures tested. The water tension of $-80 \mathrm{kPa}$ tended to show the highest percent of germination, although it did not differ from $-8,-300$ and $-1,500 \mathrm{kPa}$ water tensions. The microsclerotia did not germinate when sand dried at air temperature; however, they germinated at water tension of 0 $\mathrm{kPa}$, although at a lower rate than the germination at the other water tensions studied. The isolated effects of water tension on the microsclerotia germination were important only when the extremes values of this factor were considered.

\section{INTRODUÇÃO}

Macrophomina phaseolina (Tassi) Goidanich causa podridão de raízes, caule, ramos e frutos em diferentes espécies botânicas, sendo responsável por incitar doenças em mais de

\footnotetext{
*Parte da Tese de Doutorado do primeiro autor. Universidade Estadual Paulista (1996).
}

500 culturas econômicas (Sinclair \& Backman, 1989).

A persistência de fungos habitantes de raízes e de solo na ausência de um hospedeiro ou em condições ambientais desfavoráveis é comumente prolongada pela formação de estruturas de resistência. Segundo Smith (1969), $M$. phaseolina sobrevive na forma de microescleródios escuros, multicelulares e de tamanho variável, produzidos em grande número no tecido hospedeiro infetado. 
O estudo das condições que podem favorecer uma epifitotia é de grande importância para a agricultura econômica, principalmente se esse patógeno é um organismo capaz de sobreviver por muito tempo sob condições adversas, como é o caso do fungo $M$. phaseolina no solo. Estudos sobre a germinação de escleródios fúngicos têm sido publicados em pequeno número e, em relação a $M$. phaseolina, apesar dos trabalhos já realizados sobre sua fisiologia, pouca importância tem sido dada à observação de seus microescleródios no solo (Collins et al., 1991).

A temperatura é um dos mais importantes fatores físicos ambientais que interferem na fisiologia dos fungos fitopatogênicos, podendo exercer uma influência direta na sobrevivência dos escleródios, devido ao seu efeito sobre a germinação, influenciando, portanto, na sobrevivência de M. phaseolina (Lohda et al., 1990). Abundante produção de microescleródios desse fungo foi produzida a temperatura de $30{ }^{\circ} \mathrm{C}$, enquanto sua inativação térmica foi obtida em discos de celofane esterilizados a temperatura de $50{ }^{\circ} \mathrm{C}$ por um período de $2 \mathrm{~h}$ (Das, 1988). Microescleródios de $M$. phaseolina sobreviveram por mais de $72 \mathrm{~h}$ em BDA a $45^{\circ} \mathrm{C}$, porém foram inviabilizados quando expostos à temperatura de $55{ }^{\circ} \mathrm{C}$ por $24 \mathrm{~h}$, enquanto a $50{ }^{\circ} \mathrm{C}$ apenas $11 \%$ dos microescleródios permaneceram viáveis após 21 dias (Sheik \& Ghaffar, 1987).

O conteúdo de água no solo é outro importante fator do ambiente a afetar a sobrevivência de M. phaseolina. Estudos realizados por Banerjee et al. (1982) revelaram que a umidade, mas não a saturação, foi necessária para a germinação dos microescleródios de $M$. phaseolina. Foi verificado que, em geral, os patógenos que infetam raízes, incluindo $M$. phaseolina, são favorecidos por baixo teor de umidade no solo (Vale \& Zambolim, 1996). Collins et al. (1991) observaram inibição na germinação de microescleródios de $M$. phaseolina em solos arenosos com tensão de água de $10 \mathrm{kPa}$, atribuindo tal inibição à redução no conteúdo de $\mathrm{O}_{2}$ do solo. Esses autores verificaram também uma maior taxa de germinação em um solo arenoso em comparação a outro siltoso. Gangopadhyay et al. (1982) verificaram que a percentagem de germinação de microescleródios de $M$. phaseollina foi reduzida quando a umidade $(\theta)$ foi de $5 \% \mathrm{em}$ solos com densidades de $1,30 \mathrm{~g} / \mathrm{cm}^{3}$ e de $1,56 \mathrm{~g} / \mathrm{cm}^{3}$, mas não em densidade de $1,15 \mathrm{~g} / \mathrm{cm}^{3}$. Em umidade mais elevada $(\theta=15 \%)$, ocorreu maior germinação de microescleródios, exceto quando o solo foi mais compactado. Estudos de Kendig et al. (2000) indicaram que o manejo da água pode ter um efeito significativo sobre a colonização de raízes da soja (Glycine max L.) por M. phaseolina.

Em face da importância epifitotiológica dos microescleródios de $M$. phaseolina para diversas culturas econômicas e, ainda, devido à relativa escassez de pesquisas publicadas a respeito da germinação de suas estruturas de sobrevivência, realizou-se este trabalho, o qual objetivou investigar o efeito conjunto da temperatura e da tensão de água em substrato de areia sobre a germinação dos microescleródios do fungo.

\section{MATERIAL E MÉTODOS}

O isolado de $M$. phaseolina empregado na realização deste trabalho, FE-2084, foi obtido da micoteca do Laboratório de Fitopatologia do Departamento de Defesa Fitossanitária da Faculdade de Ciências Agrárias da UNESP, Campus de Botucatu, SP. Quando inoculado indiretamente, através de contaminação artificial do substrato, em feijoeiro (Phaseolus vulgaris L.), 'Carioca', o FE-2084 causou o tombamento das mudinhas. A metodologia para a produção dos microescleródios, bem como para o preparo da areia que serviu de substrato de germinação para essas estruturas foi baseada no trabalho de Viana \& Souza (1997).

Com base nos resultados obtidos em trabalho anterior (Viana \& Souza,1997), selecionaram-se temperaturas em torno de $30^{\circ} \mathrm{C}$, as quais pudessem simular as condições em que $M$. phaseolina fosse encontrada em solos naturais. Em uma primeira etapa (Experimento 1), foram testadas as temperaturas de $24{ }^{\circ} \mathrm{C}, 27{ }^{\circ} \mathrm{C}, 30{ }^{\circ} \mathrm{C}, 33{ }^{\circ} \mathrm{C}$ e $36{ }^{\circ} \mathrm{C}$, para interagir com dois níveis de tensões de água. $\mathrm{O}$ primeiro nível, correspondendo ao solo seco, com tensão de água infinita $\left(\psi_{\mathrm{m}}=\infty\right)$ e, o segundo, com tensão de água correspondente ao teórico ponto de murcha permanente-PMP $\left(\psi_{\mathrm{m}}=-1500 \mathrm{kPa}\right)$, ambos os pontos obtidos da curva característica de água na areia (Viana \& Souza, 1997), ajustada segundo o modelo de Genutchen (1980).

Na segunda etapa (Experimento 2), empregaram-se as duas temperaturas que tenderam a favorecer um maior percentual de germinação de microescleródios no Experimento 1, envolvendo as temperaturas de $30{ }^{\circ} \mathrm{C}$ e 33 ${ }^{\circ} \mathrm{C}$. Essas temperaturas foram postas a interagir com seis tensões de água $\left(\psi_{\mathrm{m}}\right)$ na areia: areia seca ao ar $\left(\psi_{\mathrm{m}}=\infty\right)$, $1.500 \mathrm{kPa},-300 \mathrm{kPa},-80 \mathrm{kPa},-8 \mathrm{kPa}$ e $0 \mathrm{kPa}$. As tensões de água e temperaturas na areia foram obtidas do mesmo modo que no Experimento 1. O ponto $\psi_{\mathrm{m}}=-1.500 \mathrm{kPa}$ foi selecionado como parâmetro limite mínimo, enquanto o ponto $\psi_{\mathrm{m}}=0 \mathrm{kPa}$ fixou o limite máximo de água no substrato, areia saturada. Com base nas indicações de Reichardt (1988), considerou-se capacidade de campo (CC) na areia no ponto correspondente a $\psi_{\mathrm{m}}=-8 \mathrm{kPa}$, os outros pontos foram incluídos para aumentar o espectro de avaliação do teste.

Os microescleródios empregados no Experimento 1 tinham cerca de 30 dias de idade, enquanto os empregado no Experimento 2 tinham menos de $72 \mathrm{~h}$.

A incubação dos microescleródios nas condições descritas foi realizada por um período de 24 h, após o que se efetuou a avaliação dos tratamentos através da determinação do percentual médio de estruturas germinadas em cada uma das duas membranas que compunha a parcela. Para contagem dos microescleródios germinados, efetuou-se uma simplificação do método empregado por Hinz (1991). Após a incubação, as membranas foram retiradas dos frascos e, com auxílio de pincel macio, removeram-se as partículas de areia aderidas à face externa de cada uma das duas membranas que, em seguida, foram separadas e depositadas sobre uma lâmina de vidro, com as faces contendo os microescleródios 
voltadas para cima. Em seguida, as microestruturas em cada membrana foram coradas com azul de Amann e procedeu-se a contagem dos microescleródios germinados com auxílio de microscópio ótico com aumento de 120 vezes.

O delineamento experimental para ambos os experimentos foi o inteiramente casualizado com quatro repetições, em esquema fatorial no arranjo combinatório $5 \mathrm{x}$ 2 para o Experimento 1, e 2 x 6 para o Experimento 2. Para efeito de análise, os dados obtidos nos dois experimentos foram transformados em $\sqrt{x+1}$, de modo a aproximá-los de uma curva normal. Para a identificação de diferenças entre os tratamentos, empregou-se o teste de médias de Duncan para ambos os experimentos.

\section{RESULTADOS E DISCUSSÃO}

Os dados de ambos os experimentos foram analisados através do programa SAS System versão 8.1e. A análise da variância com a aplicação do teste $\mathrm{F}$ aos dados do Experimento 1 mostrou significância apenas para o fator umidade $(\mathrm{P} \leq 0,01)$. Contudo, aplicando-se o teste de Duncan (Tabela 1), verificouse que à temperatura de $24^{\circ} \mathrm{C}$ ocorreu o menor percentual de germinação $(\mathrm{P} \leq 0,05)$, estatisticamente inferior às demais testadas. A temperatura de $33{ }^{\circ} \mathrm{C}$, apesar de proporcionar germinação estatisticamente $(\mathrm{P}>0,05)$ semelhante às temperaturas de $27^{\circ} \mathrm{C}, 30^{\circ} \mathrm{C}$ e $36^{\circ} \mathrm{C}$, mostrou uma tendência favorável a um maior percentual de germinação e um maior vigor desta, pois os microescleródios apresentaram um maior número de células germinantes.

Os dois níveis do fator tensão de água testados foram estatisticamente distintos entre si, não tendo ocorrido germinação de microescleródios em areia seca ao ar $\left(\psi_{\mathrm{m}}=\infty\right)$, o que resultou em ausência de interação entre os fatores estudados, demonstrada pela análise da variância. Nesse nível de tensão de água no substrato, a completa ausência de germinação dos microescleródios ocorreu porque a água se encontrava em um nível considerado indisponível, sugerindo existir um nível mínimo de umidade no substrato para a germinação dos microescleródios. Kendig et al. (2000) verificaram um forte efeito do estresse de água na colonização inicial de raízes de soja por M. phaseolina a partir de microescleródios. Papavizas (1977), por sua vez, já havia observado rápido declínio na sobrevivência de microescleródios de $M$. phaseolina em solo

TABELA 1 - Efeito da interação de duas tensões de água com cinco temperaturas sobre a germinação de microescleródios de Macrophomina phaseolina em areia

\begin{tabular}{lccccc}
\hline \hline \multirow{2}{*}{$\begin{array}{l}\text { Tensão de } \\
\text { água (kPa) }\end{array}$} & \multicolumn{5}{c}{ Germinação de microescleródios (\%) } \\
\cline { 2 - 6 } & $\mathbf{2 4}{ }^{\mathbf{0}} \mathbf{C}$ & $\mathbf{2 7}^{\mathbf{0}} \mathbf{C}$ & $\mathbf{3 0}{ }^{\mathbf{C}} \mathbf{C}$ & $\mathbf{3 3}{ }^{\mathbf{}} \mathbf{C}$ & $\mathbf{3 6}^{\mathbf{}} \mathbf{C}$ \\
\hline${ }^{1} \Psi_{\infty}$ & 0,0 & 0,0 & 0,0 & 0,0 & 0,0 \\
$1500 \mathrm{kPa}$ & $13,5 \mathrm{~b}$ & $22,0 \mathrm{ab}$ & $29,0 \mathrm{ab}$ & $36,7 \mathrm{a}$ & $28,5 \mathrm{ab}$ \\
\hline
\end{tabular}

Médias da mesma linha seguidas da mesma letra não diferem, estatisticamente, entre si pelo teste de Duncan a 5\% de probabilidade.

${ }^{1}$ Areia seca ao ar. seco ao ar.

O teste "F" aplicado aos dados do Experimento 2, que objetivou verificar o efeito de diferentes tensões de água na germinação dos microescleródios, bem como o efeito dessas tensões associadas a cada uma das temperaturas, isoladamente, mostrou que pelo menos uma das tensões testadas foi diferente das demais $(\mathrm{P} \leq 0,01)$. A comparação de médias dessas tensões de água pelo teste de Duncan mostrou que em areia seca ao ar $\left(\psi_{\mathrm{m}}=\infty\right)$ ocorreu o menor percentual de germinação (Tabela 2), que foi estatisticamente inferior às outras tensões testadas $(\mathrm{P} \leq 0,01)$, seguida da tensão $0 \mathrm{kPa}$, esta também estatísticamente inferior $(\mathrm{P} \leq 0,01)$ às demais, mas superior à tensão na areia seca ao ar $\left(\psi_{\mathrm{m}}=\infty\right)$.

Embora o teste " $F$ " não tenha revelado diferenças entre temperaturas e na interação dos fatores estudados (umidade e temperatura) (Tabela 2), é interessante observar que o desdobramento do fator temperatura em relação às tensões de água somente foi significativo no ponto correspondente a um teor de água considerado bom para a germinação de $M$. phaseolina, em $-8 \mathrm{kPa}$. Nesta tensão, a água se encontrava disponível sem saturar o substrato. Este é um fenômeno comum entre os seres vivos, quando um importante fator biótico está no nível ótimo, um outro lhe sucede, passando a exercer maior influência na vida do organismo.

Como Banerjee et al. (1982), neste trabalho, verificouse que a umidade foi necessária à germinação dos microescleródios de $M$. phaseolina, pois em areia seca $\left(\psi_{\mathrm{m}}=\right.$ $\infty)$ não ocorreu germinação nos dois experimentos realizados. Entretanto, no Experimento 2, condições de umidade moderada foram mais favoráveis que condições extremas, como de substrato seco ou saturado, em ambas as temperaturas testadas. A comparação das temperaturas que proporcionaram maiores percentuais de germinação no Experimento 2, em relação a diferentes tensões de água (Tabela 2) demonstrou a importância da umidade no substrato na germinação dos microescleródios de $M$. phaseolina, pois quando a tensão de

TABELA 2 - Efeito da interação de seis tesões de água com duas temperaturas sobre a germinação de microescleródios de Macrophomina phaseolina em areia

\begin{tabular}{cccc}
\hline \hline \multirow{2}{*}{$\begin{array}{c}\text { Tensão de } \\
\text { água }(\mathbf{k P a})\end{array}$} & \multicolumn{3}{c}{ Germinação de microescleródios (\%) } \\
\cline { 2 - 4 } & $\mathbf{3 0}{ }^{\mathbf{}} \mathbf{C}$ & $\mathbf{3 3}^{\mathbf{0}} \mathbf{C}$ & Médias \\
\hline${ }^{1} \Psi_{\mathrm{m}}=\infty$ & $0,0 \mathrm{cA}$ & $0,0 \mathrm{cA}$ & $0,0 \mathrm{c}$ \\
1500 & $51,5 \mathrm{aA}$ & $47,5 \mathrm{aA}$ & $49,5 \mathrm{a}$ \\
300 & $51,2 \mathrm{aA}$ & $52,5 \mathrm{aA}$ & $51,8 \mathrm{a}$ \\
80 & $51,7 \mathrm{aA}$ & $54,2 \mathrm{aA}$ & $52,9 \mathrm{a}$ \\
8 & $58,2 \mathrm{aA}$ & $45,7 \mathrm{aB}$ & $51,9 \mathrm{a}$ \\
0 & $17,0 \mathrm{bA}$ & $26,2 \mathrm{bA}$ & $21,6 \mathrm{~b}$ \\
\hline
\end{tabular}

Médias de mesma coluna, seguidas da mesma letra minúscula (umidade e umidade/temperatura), assim como médias da mesma linha seguidas da mesma letra maiúscula (temperatura/umidade), não diferem entre si pelo teste de Tukey a $5 \%$ de probabilidade.

${ }^{1}$ Areia seca ao ar 
água na areia foi reduzida até à saturação $\left(\psi_{\mathrm{m}}=0 \mathrm{kPa}\right)$, a germinação foi inibida. Resultado semelhante foi obtido por Banerjee et al. (1993) que anotaram perda de até $85 \%$ na população de microescleródios desse fungo em umidade de saturação.

Embora Dhingra \& Sinclair (1974) tenham verificado perda da viabilidade em microescleródios de M. phaseolina em solos com umidade de $100 \%$ e $80 \%$ da capacidade de campo, neste trabalho, a germinação somente foi afetada significativamente quando a tensão de água correspondeu a $0 \mathrm{kPa}$. No experimento 2, em ambas temperaturas testadas, a germinação dos microescleródios desde o teórico ponto de murcha permanente $\left(\psi_{\mathrm{m}}=-1500 \mathrm{kPa}\right)$ até o téorico ponto de capacidade de campo $\left(\psi_{\mathrm{m}}=-8 \mathrm{kPa}\right)$ foi estatisticamente semelhante, diferindo apenas na menor tensão de água $\left(\psi_{\mathrm{m}}=\right.$ $0 \mathrm{kPa}$ ). Shokes et al. (1977) recuperaram elevado percentual de microescleródios viáveis de M. phaseolina em tensões de água no solo desde $-1 \mathrm{kPa}$ até $-1150 \mathrm{kPa}$, tendo anotado queda de $35 \%$ na sobrevivência destes em solo saturado.

No Experimento 2, microescleródios submetidos à mesma tensão de água e temperatura do Experimento 1, ou seja, $-1.500 \mathrm{kPa}$ e $30^{\circ} \mathrm{C}$, tiveram percentuais de germinação diferentes. É possível que a diferença entre as idades dessas microestruturas nos dois experimentos seja o fator responsável. Contudo, acredita-se que, não a viabilidade, mas o aprofundamento da dormência ao longo do tempo seja o responsável pelo menor percentual de germinação no Experimento 1, cujos microescleródios eram mais velhos em cerca de 27 dias. Esse prazo seria muito curto para ocasionar a perda de viabilidade de uma estrutura de resistência tão eficiente.

No Experimento 2, em areia com teor de umidade correspondente à capacidade de campo $\left(\psi_{\mathrm{m}}=-8 \mathrm{kPa}\right)$, obtevese um percentual de germinação estatisticamente semelhante ao obtido na tensão mais propícia para a germinação $\left(\psi_{\mathrm{m}}=\right.$ $80 \mathrm{kPa}$ ) nas duas temperaturas testadas, embora Dhingra \& Sinclair (1974) tenham observado perda na viabilidade de microescleródios de $M$. phaseolina em solos com teores de umidade correspondentes a capacidade de campo. Contudo, Collins et al. (1991) verificaram maior percentual de germinação de microescleródios desse fungo em potencial de água do solo de $-1500 \mathrm{kPa}$, o que está de acordo com os dados obtidos neste trabalho.

É provável que diferenças nas propriedades físicas e nutricionais do solo, além da temperatura em que se realizaram os testes, sejam responsáveis por diferenças nos resultados de algumas pesquisas referentes ao efeito de teores de água na germinação de microescleródios de M. phaseolina. Isolado o efeito nutricional sobre a germinação dos microescleródios observado neste trabalho, o que pode ser verificado nos dados de Viana \& Souza (1997), que apresentam valores nulos para carbono e para nitrogênio na areia empregada como substrato, verificou-se que, em tensão de $\psi_{\mathrm{m}}=-8 \mathrm{kPa}$, uma alteração de $3{ }^{\circ} \mathrm{C}$ no substrato foi $\mathrm{o}$ bastante para proporcionar uma diferença de $21 \%$ na germinação. Verificou-se ainda, que diferenças na idade dos microescleródios empregados nos testes podem refletir-se como diferenças na germinação dessas microestruturas de resistência quando submetidas a condições semelhantes.

Constataram-se ainda, que os microescleródios de $M$. phaseolina germinaram em um percentual médio superior a $58 \%$ em areia natural em $24 \mathrm{~h}$, o que se traduz em elevada proporção, considerando que essa estrutura de resistência do fungo pode apresentar dormência de duas naturezas, constitutiva e exógena. Os resultados desse estudo fazem concluir que o efeito do fator tensão de água sobre a germinação dos microescleródios de $M$. phaseolina somente foi importante quando esta tensão foi muito elevada $\left(\psi_{\mathrm{m}}=\infty\right)$ ou muito baixa $\left(\psi_{\mathrm{m}}=0 \mathrm{kPa}\right)$.

A manipulação do ambiente do solo, por vezes, é a única medida possível de ser efetuada para reduzir ou controlar uma doença. Diversos autores conseguiram redução da podridão de raízes em algumas culturas atacadas por $M$. phaseolina pelo emprego de práticas culturais que interferiam com a temperatura e a umidade do solo (Mihail et al., 1989).

O conhecimento dos efeitos da interação temperaturatensão de água no substrato sobre a germinação de estruturas de resistência de patógenos do solo é de elevado alcance prático, quando se pensa no controle cultural de doenças induzidas por esses microrganismos. Portanto, a solarização, prática que interfere, conjuntamente, com a temperatura e a umidade no ambiente do solo pode se constituir em excelente alternativa de controle de doenças causadas por M. phaseolina, principalmente em regiões de clima semelhantes ao do Nordeste brasileiro.

\section{REFERÊNCIAS BIBLIOGRÁFICAS}

BANERJEE, S., MUKHERJEE, B. \& SEN. C. Fungistasis and germination patterns of sclerotia of Macrophomina phaseolina (Tassi) Goid. in different soils. Indian Journal of Microbiology 22:190-193. 1982.

BANERJEE, S., MUKHERJEE, B. \& SEN, C. Survival of mycelia and sclerotia of Macrophomina phaseolina in soil: influence of moisture and temperature. Indian Journal of Plant Patholology 1:20-23. 1993.

COLLINS, D.J., WYLLIE, T.D. \& ANDERSON, S.H. Biological activity of Macrophomina phaseolina in soil. Soil Biology and Biochemestry 23:495-496. 1991.

DAS, N.D. Effect of different sources of carbon, nitrogen and temperature on the growth and sclerotial production of Macrophomina phaseolina (Tass.) Goid. causing root rot / charcoal rot disease of castor. Indian Journal of Plant Pathology 6:97-98. 1988.

DHINGRA, O.D. \& SINCLAIR, J.B. Effect of soil moisture and carbon: nitrogen ratio on survival of Macrophomina phaseolina in soybean stems in soil. Plant Disease Reporter 58:1034-1037. 1974.

GANGOPADHYAY, S., WYLLIE, T.D. \& TEAGUE, W.R. Effect of bulk density and moisture content of soil on the survival of Macrophomina phaseolina. Plant and Soil 68:241-247. 1982.

GENUTCHEN, M.T. A closed-form equation for prediting the hidraulic conductivity of unsatured soils. Soil Science Society America Journal 44:892-898. 1980. 
HINZ, R.H. Germinação de microconídios de Fusarium oxyxporum f. sp. cubense (E.F. Smith) Snyder \& Hansen em areia incorporada com manipueira. (Tese de Mestrado). Botucatu, UNESP. 1991.

KENDIG, S.R., RUPE,J.C. \& SCOTT,H.D. Effect of irigation and soil water stress on densities of Macrophomina phaseolina in soil and roots of two soybeans cultivars. Plant Disease 84:895900. 2000

LODHA, S., MATHUR, B.K. \& SOLANKI, K.R. Factors influencing population dynamics of Macrophomina phaseolina in arid soils. Plant and Soil 125:75-80. 1990.

MIHAIL, J.D., ORUM, T.V. \& ALCORN, S.M., RAY, D. Macrophomina phaseolina in the Sonoran Desert. Canadian Journal of Botany 67:76-82. 1989.

PAPAVIZAS, G.C. Some factors affecting survival of sclerotia of Macrophomina phaseolina in soil. Soil Biology and Biochemistry 9:337-341. 1977.

REICHARDT, K. Capacidade de campo. Revista Brasileira de Ciência do Solo 12:211-216. 1988.
SHEIKH, A.H. \& GHAFFAR, A. Time-temperature relationships for the inactivation of sclerotia of Macrophomina phaseolina. Soil Biology and Biochemistry 19:313-315. 1987.

SHOKES, F.M., LYDA, S.D. \& JORDAN, W.R. Effect of water potential on the growth and survival of Macrophomina phaseolina. Phytopathology 67: 239-241. 1977.

SINCLAIR, J.B. \& BACKMAN, P.A. Compendium of soybean diseases. 3. ed. St. Paul: American Phytopathological Society, 1989.

SMITH, W.H. Germination of Macrophomina phaseolina sclerotia as effected by Pinus lambertiana root exudate. Canadian Journal of Microbiology 15:1387-1391. 1969.

VALE, F.X.R. \& ZAMBOLIM, F. Influência da temperatura e da umidade nas epidemias de doenças de plantas. Revisão Anual de Patologia de Plantas 4:149-207. 1996.

VIANA, F.M.P. \& SOUZA, N.L. Efeito da temperatura e tensão de água do substrato na germinação de microescleródios de Macrophomina phaseolina. Summa Phytopathologica 23:236239. 1997. 